Pacific Journal of Mathematics

Vol. 5, No. 3

November 1955 


\title{
ON FACTOR FUNCTIONS
}

\author{
R. E. EDW ARDS
}

0. Introduction. The object of this paper is to illustrate by means of a few selected examples the application of abstract but simple methods to the study of factor functions. The methods have a considerably wider range of application then is explicitly covered here: in particular, it applies to functional transformation other than that of Fourier.

The factor problem is understood in the following sense. $G$ will denote throughout a locally compact and abelian group; $E$ and $F$ will be two topological vector spaces of functions, measures or distributions on $G$ for which the Fourier transformation is suitably defined. The transform of $f$ is denoted generally by $\hat{f}$. A function $\phi$ on $\hat{G}$, the group dual to $G$, is said to be a (Fourier) factor of class $(E, F)$ if and only if $\phi \cdot \hat{f}$ is the transform of some $g \in F$ each time that $f \in E$. In all cases we have in mind; $E$ and $F$ are each invariant under the translations by group elements $t_{x}(x \in G)$, and in many such cases it is convenient to subordinate the factor problem to that of finding a representation theorem for a general continuous linear mapping $u$ of $E$ into $F$ which commutes with translations. The class of such mappings is denoted by $L_{t}(E, F)$, the notation $L(E, F)$ being reserved for the set of all continuous linear mappings of $E$ into $F$.

The formal relationship between the two problems is expressed as follows. If $\phi$ is a factor of class $(E, F)$, let $u$ be the linear mapping of $E$ into $F$ which is defined by agreeing that $g=u(f)$ is to signify that $\hat{g}=\phi \cdot \hat{f}$. The continuity of $u$ is usually a consequence of the "closed graph theorem", whilst the fact that $u$ commutes with translations is a consequence of the way that translation effects the Fourier transformation (multiplication by characters of $G$ ). On the other hand it is not always easy to show that every $u \in L_{t}(E, F)$ is derivable in this manner from a factor function of class $(E, F)$.

In most of the applications dealt with below, $E$ and $F$ are both Fréchet spaces. The one general property of these spaces we use is the weak relative compacity of weakly bounded subsets of the dual of such a space. 
1. Mappings of class $L_{t}\left(L^{1}, L^{p}\right)$. As usual, the translate $t_{a} f$ of a function $f$ corresponding to the group element $a$ is defined by

$$
t_{a} f(x)=f(x+a)
$$

for $x \in G$. Suppose that the exponent $p$ is suitably restricted if $G$ is non-compact; then a factor function $\phi$ of class $\left(L^{1}, L^{p}\right)$ gives rise, in a manner already described, to a mapping $u \in L_{t}\left(L^{1}, L^{p}\right)$. This is one instance where the continuity of $u$ is a direct consequence of the "closed graph theorem". Having solved the representation problem, an inverse Fourier transformation leads back to the form of $\phi$. The centre of the programme is thus to establish

THEOREM 1. Let $1 \leq p \leq \infty$. The mappings $u \in L_{t}\left(L^{1}, L^{p}\right)$ are those and only those of the form

$$
u(f)=\mu * f
$$

where $\mu$ depends only upon $u$ and is such that

(i) $\mu$ is a bounded Radon measure on $G$, if $p=1$, and

(ii) $\mu \in L^{p}$ if $1<p \leq \infty$.

Proof. In either case there is no difficulty in varifying that (1.1) defines $u$ as a member of $L_{t}\left(L^{1}, L^{p}\right)$ each time that $\mu$ is restricted according to (i) or (ii). We remark that the convolution on the right of $(1.1)$ is most conveniently regarded as an abstract integral $\int_{G}\left(t_{-x} f\right) d \mu(x)$ interpreted in either a strong or a weak sense.

To prove the converse, we begin by remarking that if $k * f$ is interpreted for $k, f \in L^{1}$ as an abstract integral, then $u \in L_{t}\left(L^{1}, L^{p}\right)$ implies the relation

$$
u(k * f)=u(k) * f .
$$

We now let $k$ vary along an "approximate identity in $L^{1 "}$. By this we mean a directed family $\left(k_{i}\right)$ of positive functions in $L^{1}$ such that

$$
\int_{G} k_{i}(x) d x=1
$$

for all $i$ and $\lim _{i}\left(k_{i} * f\right)=f$ in mean for each $f$ in $L^{1}$; the latter condition can be satisfied if, for example, it is arranged that $k_{i}$ has its support contained in a neighborhood $U_{i}$ of 0 in $G$, the $U_{i}$ forming a decreasing directed system and a neighborhood base at 0 in $G$. From (1.2) there results 


$$
u(f)=\lim _{i}\left\{u\left(k_{i}\right) * f\right\}
$$

by virtue of the continuity of $u$. The continuity of $u$ ensures also that the $\mu_{i}=$ $u\left(k_{i}\right)$ fall into a norm-bounded subset of $L^{P}$.

If $p>1$ then $L^{p}$ is the dual of $L^{p^{\prime}}\left(1 / p+1 / p^{\prime}=1\right)$ and the norm-bounded subsets of $L^{p}$ are therefore weakly relatively compact. This, combined with (1.3), leads to the conclusion that there is a $\mu$ which is a weak limiting point of the directed family $\left(\mu_{i}\right)$ and for which $u(f)=\mu * f$ at least for all continuous $f$ with compact supports, hence by continuity for all $f \in L^{1}$. If $G$ satisfies the first countability axiom then the directed family $\left(k_{i}\right)$ may be taken to be a denumerable sequence and the details of the argument are quite clear. In the contrary case, although the principle is exactly the same, a few technical details must be attended to. These details present but little intrinsic interest and are accordingly omitted.

If $p=1$ then the above argument breaks down since the norm-bounded subsets of $L^{1}$ are not all weakly relatively compact. So we imbed $L^{1}$ in the space $M^{1}$ of bounded Radon measures on $G$, the latter being looked upon as the dual of the space $C_{0}$ of continuous functions on $G$ which vanish at infinity equipped with the uniform norm. From this point onwards the argument proceeds much as before.

REMARKS. (i) If $G$ is compact then the translation of Theorem 1 into terms of factor functions is straightforward. In the contrary case complications arise when $p>2$ due to which of several possible senses assignable to the Fourier transformation is adopted. Such cases lead one to regard the representation problem as occupying the more fundamental position in general.

(ii) The case $p=1$ of Theorem 1 , expressed in terms of factor functions, is in the literature at any rate for the cases in which $G$ is the circle group or the real line: see Zygmund [5, p. 101 ] and Hille [2, p. 362].

Factors of class $\left(M^{1}, M^{1}\right)$. We are led to consider two topologies on $M^{1}$, namely the normed topology regarding $M^{1}$ as the dual of $C_{0}$, and the corresponding weak topology $\sigma\left(M^{1}, C_{0}\right)$. When we wish to call attention to the distinction we shall use the notations $M_{n}^{1}$ and $M_{w}^{1}$ to denote the corresponding topological vector spaces.

The solution of the representation problem for $L_{t}\left(M_{w}^{1}, M_{w}^{1}\right)$ is simpler than that for $L_{t}\left(M_{n}^{1}, M_{n}^{1}\right)$. If $s$ stands for $n$ or $w$, the crux in either proof is to show that any $u \in L_{t}\left(M_{s}^{1}, M_{s}^{1}\right)$ satisfies 


$$
u(\alpha * \beta)=u(\alpha) * \beta
$$

for all $\alpha, \beta \in M^{1}$. Once this established, the representation follows immediately on choosing $\alpha$ to be the Dirac measure at the origin of G. Equation (1.4) is satisfied automatically by any $u$ derived from a factor function of class $\left(M^{1}, M^{1}\right)$ and this problem is therefore closed; see also Zygmund [5, p. 101] for the circle group.

If $u \in L_{t}\left(M_{w}^{1}, M_{w}^{1}\right)$ then (1.4) can be established without difficulty by means of the representation

$$
\alpha * \beta=\int_{G}\left(t_{-x} \alpha\right) d \beta(x)
$$

of $\alpha * \beta$ by means of a vector-valued integral relative to the weak topology. If $w$ is replaced by $n$ then (1.5) is no longer valid for all $\alpha$ and $\beta$ because for general $\alpha \in M^{1}$ the vector-valued function $x \rightarrow t_{-x} \alpha$ is not even measurable for $\sigma\left(M_{n}^{1}, M_{n}^{1 \prime}\right)$, far less strongly measurable. The representation problem for mappings of class $L_{t}\left(M_{n}^{1}, M_{n}^{1}\right)$ is left open for further discussion.

Factors of class (\&, \&). Making another change of direction, one can take $G=R^{n}$ and consider the non-classical factor problem associated with Schwartz's space $\&[4$, Tome II, Chapitre VII]. Here again the factor problem can be conveniently' subordinated to the representation problem for $L_{t}(\infty, \infty)$. We will state, and sketch the proof of, the appropriate theorem because, although it is like that of Theorem 1 in principle, the details are some what more complicated.

THEOREM 2. The mappings $u \in L_{t}(\$, \S)$ are those and only those of the form

$$
u(f)=\mu * f
$$$$
(f \in \infty) \text {, }
$$

where $\mu$ depends only upon $u$ and is a rapidly decreasing distribution.

Proof. As before, it is easy to see that (1.6), with $\mu \in O_{C}^{\prime}$, defines $u$ as a member of $L_{t}(\$, \$)$. For $u$, thus defined, is obviously linear and commutes with translations; that it is continuous is perhaps most easily seen by an application of the Fourier transformation, effecting a topological automorphism of $\&$, combined with the observation that $\hat{\mu} \in O_{M}$ whenever $\mu \in O_{C}^{\prime}$. These facts are all to found in Schwartz's treatise.

Conversely, assume that $u \in L_{t}(\&, \S)$. If $k, f \in \varnothing$ one can write 


$$
k * f=\int_{G}\left(t_{-x} k\right) \cdot f(x) d x
$$

the function $x \rightarrow t_{-x} k \cdot f(x)$ mapping $G$ into the Fréchet space \& being continuous and rapidly decreasing. Since $u$ is continuous and commutes with translations, it follows that

$$
u(k * f)=u(k) * f
$$

We now let $k$ vary along an "approximate identity" formed of a denumerable sequence $\left(k_{i}\right)$, each $k_{i}$ being "smoothed" if necessary so as to belong to $\&$. Then

$$
u(f)=\lim _{i}\left(\mu_{i} * f\right)
$$

in the sense of $\diamond$, where $\mu_{i}=u\left(k_{i}\right) \in \varnothing$. Now regard the $\mu_{i}$ as distributions, elements of \& : the last equation shows that the sequence $\left(\mu_{i}\right)$ is weakly bounded in $\&$, so that, \& being a separable Fréchet space, one may assume that $\mu_{i} \longrightarrow \mu$ weakly in $\&^{\prime}$. Actually, since $\&$ is a Montel space, we may even assume that $\mu_{i} \rightarrow \mu$ strongly in $\delta^{\prime}$, but this refinement is not necessary to our argument. The weak convergence $\mu_{i} \rightarrow \mu$ is already enough to arrange that $\mu_{i} * f \longrightarrow \mu * f$ pointwise for each $f \in \varnothing$. Hence we conclude that (1.6) holds.

The proof is however not yet complete for we wish to show that $\mu$ is not merely an element of $\varnothing^{\prime}$, but is in addition rapidly decreasing, that is, $\mu \in O_{C}^{\prime}$. However if we put $T \in \hat{\mu}$ then (1.6) shows that $T$ is a distribution $\in \&^{\prime}$ such that the multiplicative product $T \cdot F \in \&$ each time that $F \in \&$. Introduce the linear mapping $w$ of $\&$ into itself defined by $w(F)=T \cdot F$. Once it is shown that $w$ is continuous, an easy argument leads to the conclusion that $T$, which is plainly forced to be an indefinitely differentiable function, has the property that it and each of its derivatives is majorised by some polynomial (depending perhaps on the derivative in question). That is, $T$ must belong to $O_{M}$ and so $\mu$ must belong $O_{C}^{\prime}$.

Thus we are reduced to showing that $w$ is continuous from $\&$ into itself. To do this, introduce a sequence $\left(h_{n}\right)$ of functions extracted from the space $D$ of indefinitely differentiable functions with compact supports such that

$$
h_{n}(x) \rightarrow 1, D^{p} h_{n}(x) \rightarrow 0 \quad(p=1,2, \cdots ; D=d / d x),
$$

in each case boundedly and uniformly on compact sets. Put $w_{n}(F)=h_{n} T F$ for 
$F \in \bigotimes$. The hypothesis that $T F \in \varnothing$ implies that $w_{n}(F) \rightarrow w(F)$ in the sense of $\&$ for each $F$. Since $\&$ is a Fréchet space, the Banach-Steinhaus theorem affirms the continuity of $w$ once that of each $w_{n}$ is granted. But the continuity of $w_{n}$ is obvious since $h_{n} \in \mathbb{D}$ and $T$ is an indefinitely differentiable function. This completes the proof.

Corollary. A function $\phi$ is a factor of class (\$, \$), if and only if it belongs to $O_{M}$.

REMARK. Theorem 2 is simultaneously a special case and a refinement of a theorem of Schwartz [4, Tome II, pp. 19 and 53].

2. The adjoint process. This process is well to the fore in standard literature, as is witnessed by [5, p. 103] and Kaczmarz-Steinhaus [3, p. 223]. We illus trate by applying it to the results obtained in $\S 1$ above.

THEOREM 3. Let $1 \leq q<\infty$. The mappings $u \in L_{t}\left(L^{q}, L^{\infty}\right)$ are precisely those having the form

$$
u(f)=\mu * f
$$

where $\mu \in L^{q^{\prime}}$ depends only upon $u$.

Proof. A trivial application of Hölder's inequality shows that (2.1) defines $u$ as an element of $L_{t}\left(L^{q}, L^{\infty}\right)$ whenever $\mu \in L^{q^{\prime}}$ ( and this even when $q=\infty$ ). Conversely, suppose that $u \in L_{t}\left(L^{q}, L^{\infty}\right)$ with $q$ finite. Let $v$ be the adjoint of $u . v$ is a continuous linear mapping of $\left(L^{\infty}\right)^{\prime}$ into $\left(L^{q}\right)^{\prime}=L^{p}$ with $p=q^{\prime}$. Now restrict $v$ to the subspace $L^{1}$ of $\left(L^{\infty}\right)^{\prime}$, the result being clearly a mapping of class $L_{t}\left(L^{1}, L^{p}\right)$. Since here $p=q^{\prime}>1$, Theorem 1 shows that there is a fixed $\nu \in L^{p}=L^{q^{\prime}}$ such that $v(F)=\nu * F$ for $F \in L^{1}$. Using the definition of $v$ as the adjoint of $u$, the theorem follows with $\mu=\breve{\nu}$, the reflection of $\nu$ in the origin of $G$.

The excluded case $q=\infty$ of Theorem 2. The above proof breaks down when $q=\infty$ because $L^{1}$ is not reflexive. However, if $L_{w}^{\infty}$ denotes $L^{\infty}$ equipped with the weak topology $\sigma\left(L^{\infty}, L^{1}\right)$, it is relatively easy to show that the mappings $u \in L_{t}\left(L_{w}^{\infty}, L_{w}^{\infty}\right)$ are precisely those of the form $(2.1)$ with $\mu$ ranging over $M^{1}$.

When one comes to apply this result to the problem of factor functions of class $\left(L^{\infty}, L^{\infty}\right)$ over a compact $G$, one has to show that the mapping $u$ corresponding to a factor $\phi$ is weakly continuous. This cannot now be effected by 
simple appeal to the "closed graph theorem". Instead one can apply the following general but simple lemma which has some independent interest.

Lemma. Let $E$ and $F$ be locally convex spaces, $E$ being complete. Let $u$ be a linear mapping of $E^{\prime}$ into $F^{\prime}$ such that

(i) $u$ transforms equicontinuous subsets of $E^{\prime}$ into relatively weakly compact subsets of $F^{\prime}$, and

(ii) for any equicontinuous subset $Q$ of $E$ : the graph of the restriction of $u$ to $Q$ is weakly closed in $E^{\prime} \times F^{\prime}$.

Then $u$ is weakly continuous from $E^{\prime}$ into $F^{\prime}$.

Proof. By a theorem announced recently by Grothendieck [1], it is enough to show that the restriction of $u$ to any equicontinuous subset $Q$ of $E^{\prime}$ is weakly continuous. Since $u(Q)$ is, by $(i)$, weakly relatively compact in $F^{\prime}$, and since a filter on a compact space is convergent provided it has at most one adherence point, one is reduced to proving the following. If $\Phi$ is a filter on an equicontinuous subset $Q$ of $E^{\prime}$ which converges weakly to 0 , then 0 is the only possible weak adherence point of $u(\Phi)$. But this is ensured by (ii).

To apply the lemma to the case in hand, we take $E=F=L^{1}$ and $u$ to be the mapping of $L^{\infty}$ into itself defined by the factor function $\phi$. The "closed graph theorem" itself shows that $u$ is continuous for the normed topology on $L^{\infty}$, and hence that $(i)$ is satisfied. It remains to verify (ii). For this it suffices to show that if a norm-bounded directed family $\left(f_{i}\right)$ converges to 0 weakly in $L^{\infty}$ and $g_{i}=u\left(f_{i}\right)$ converges weakly to $g$ in $L^{\infty}$, then $g$ is necessarily 0 . But if $h \in L^{1}$ has an absolutely convergent Fourier transform $\hat{h}$ one has

$$
\left\langle h, g_{i}\right\rangle=\sum_{\hat{x}} \hat{h}(-\hat{x}) \hat{g}_{i}(\hat{x})=\sum_{\hat{x}} \hat{h}(-\hat{x}) \phi(\hat{x}) \hat{f}_{i}(\hat{x})
$$

here

$$
\sum_{\hat{x}}|\hat{h}(-\hat{x}) \phi(\hat{x})|<+\infty
$$

and $\lim _{i} \hat{f}_{i}(\hat{x})=0$ boundedly. So, by the principle of dominated convergence, $\langle h, g\rangle=\lim _{i}\left\langle h, g_{i}\right\rangle=0$. This being true for all $h \in L^{1}$ of the specified type, it follows that $\mathrm{g}=0$.

To sum up, we have shown that a function $\phi$ on the dual of a compact $G$ is 
a factor of class $\left(L^{\infty}, L^{\infty}\right)$ if and only if it is the transform of some $\mu \in M^{1}$. For the circle group the result is known; see $[5, \mathrm{p} .101]$.

Mappings of class $L_{t}\left(C_{0}, C_{0}\right)$. Let $u \in L_{t}\left(C_{0}, C_{0}\right)$ and let $v$ be its adjoint. $v$ is a linear mapping of $M^{1}$ into itself which is plainly continuous for the normed topology; equally plainly it commutes with translations. The defining equation

$$
\langle f, v(\alpha)\rangle=\langle u(f), \alpha\rangle
$$

shows that $v$ is even continuous for the weak topology $\sigma\left(M^{1}, C_{0}\right)$. By what has been said about such mappings in $\S l$, it follows that $v$, and therefore also $u$, is represented as convolution with an element of $M^{1}$. The converse is trivial.

When $G$ is compact, one has incidentally the solution of perhaps the oldest of all factor problems to be studied, namely that of class $(C, C)$ over the circle group.

Mappings of class $L_{t}\left(\bigotimes^{\prime}, \wp^{\prime}\right)$. The adjoint process applies at once to Theorem 2 and gives the representation theorem for mappings $u$ of class $L_{t}\left(\bigotimes^{\prime}, \bigotimes^{\prime}\right)$. Here it is immaterial whether we equip $\&^{\prime}$ with its weak or strong topology as the dual of $\$$.

3. The "convexity theorem" of M. Riesz. The simple methods laid out above do not of course yield the solution of the factor problem for $\left(L^{p}, L^{q}\right)$ for general exponent-pairs $(p, q)$. But we remark that a solution of the representation problem for two exponent-pairs implies that for a whole range of exponent-pairs by virtue of the "convexity theorem" of Marcel Riesz. Denote by $K$ the vector space of all continuous functions on $G$ having compact supports, and by $K^{p}$ the normed vector space obtained by equipping $K$ with the norm induced upon it by $L^{P}$. We shall be concerned only with finite values of $p$, in which case $K^{p}$ is dense in $L^{p}$. If $E$ is any complete translation-invariant topological vector space, one can identify $L_{t}\left(K^{P}, E\right)$ and $L_{t}\left(L^{P}, E\right)$ since each mapping of the first class has a unique continuous extension into one of the second. This reduction is made so that all the mappings we consider in this section have a common domain of definition, namely the vector space $K$.

Suppose now that $1 \leq p_{1}, p_{2}<\infty$ and $1 \leq q_{1}, q_{2} \leq \infty$, whilst the mapping $u$ belongs at once to $L_{t}\left(K^{p_{1}}, L^{q_{1}}\right)$ and to $L_{t}\left(K^{p_{2}}, L^{q_{2}}\right)$. The "convexity theorem" affirms that $u$ is continuous from $K^{p}$ into $L^{q}$ for any pair $(p, q)$ for which there is a number $\theta$ between 0 and 1 such that 


$$
\frac{1}{p}=\frac{1-\theta}{p_{1}}+\frac{\theta}{p_{2}} \text { and } \frac{1}{q}=\frac{1-\theta}{q_{1}}+\frac{\theta}{q_{2}} .
$$

$u$, or rather a uniquely-determined extension thereof, will belong to $L_{t}\left(L^{p}, L^{q}\right)$ for any such exponent-pair $(p, q)$. Needless to say, the corresponding factor function is completely determined by the restriction of $u$ to $K$.

In this way the "convexity theorem", combined with the adjoint process, yields simple geometrical properties of the set of points $(1 / p, 1 / q)$ of the plane $R^{2}$ corresponding to those exponent-pairs $(p, q)$ for which a given function on $G$ is a factor of class $\left(L^{p}, L^{q}\right)$ (or of the corresponding exponent-pairs linked with a given mapping $u$ ).

4. Other classes of mappings for the circle group. In this final section, $G$ will denote the circle group and $\hat{G}$ therefore the additive group of integers. We can think of $G$ as the real line modulo $2 \pi$ and thus talk in terms of ordinary Fourier series. Besides the spaces $L^{p}$ we shall consider the spaces $C^{(m)}$ of continuous functions on $G$ (that is, periodic functions on the line) having at least $m$ continuous derivatives, or having derivatives of all orders if $m$ is $\infty$. If $m$ is finite, $C^{(m)}$ is made into a Banach space by equipping it with the norm

$$
N_{m}(f)=\sup _{\substack{0 \leq r \leq m \\ x}}\left|D^{r} f(x)\right| \quad(D=d / d x) .
$$

$C^{(\infty)}$ is a Fréchet space for the topology defined by the norms $N_{m}$ with $m=$ $0,1,2, \cdots$.

Consider first the classes $L_{t}\left(C^{(m)}, C^{(n)}\right)$. The case in which $m$ and $n$ are both finite is obviously reducible to the case $m=n=0$, and this has been dismissed in $\$ 2$ above. If $m<\infty$ and $n=\infty$ then, the same sort of reduction allows one to assume that $m=0, n=\infty$; a similar remark applies to the case $m=\infty, n<\infty$. Both of these cases are easily solved. There remains only the case in which $m=n=\infty$. This case may be dealt with as in the proof of Theorem 2, the details being much simpler than there. Alternatively one can utilize the isomorphism of $C^{(\infty)}$ with the space of all rapidly decreasing two-way infinite sequences $s=\left(s_{n}\right)$, the isomorphism being established by the Fourier transformation and the sequence space being equipped with the norms

$$
s \rightarrow \sum_{n=-\infty}^{\infty}(1+|n|)^{m}\left|s_{n}\right| \quad(m=0,1,2, \cdots)
$$


so as to appear as a Fréchet space.

We now proceed to discuss the classes $L_{t}\left(L^{p}, C^{(m)}\right)$. The cases corresponding to pairs $(p, m)$ excluded from the general theorem will be discussed briefly thereafter.

Using the language of (periodic) distributions [4, Tome II, Chapitre VII], it is clear that the mapping $u$ defined by

$$
u(f)=\mu * f
$$

belongs to $L_{t}\left(L^{p}, C^{(m)}\right)(1 \leq p \leq \infty, 0 \leq m \leq \infty)$ each time that $\mu$ is a distribution such that $D^{m} \mu$ is a function $\in L^{p}$ if $m<\infty$, or such that $D^{r} \mu \in L^{p}$ ' for every finite $r$ if $m=\infty$. As a partial converse we prove.

THEOREM 4. If $p$ and $m$ are finite, any mapping $u \in L_{t}\left(L^{p}, C^{(m)}\right)$ has the form (4.1), wherein $\mu$, depending solely upon $u$, is a periodic distribution such that $D^{m} \mu \in L^{p}$ :

Proof. Retracing the ideas in the proofs of Theorems 1 and 2, take an "approximate identity" $\left(k_{i}\right)$ formed of periodic functions with derivatips of all orders, put $\mu_{i}=u\left(k_{i}\right) \in C^{(m)}$, and deduce that

$$
u(f)=\lim _{i}\left(\mu_{i} * f\right) \text { in } C^{(m)}
$$

for each $f \in L^{p}$. Of course, $\left(k_{i}\right)$ may be assumed to be a denumerable sequence. Let $r$ be any integer between 0 and $m$ inclusive. Equation (4.2) shows then that the functions $D^{r} \mu_{i}$ with $i$ varying fall into a weakly bounded subset of $L^{p}$ : Since $p$ is finite, it follows that for each $r$ there exists a function $\mu^{(r)} \in L^{p}$ ' which is a weak limiting point in $L^{p}$ of the sequence $\left(D^{r} \mu_{i}\right)$. Actually, since $L^{p}$ is separable, one may assume that by extraction of a subsequence it has been arranged that $D^{r} \mu_{i} \rightarrow \mu^{(r)}$ weakly in $L^{p^{\prime}}$ as $i \rightarrow \infty$, and this for each relevant $r$. Putting $\mu=\mu^{(0)}$, it is easily seen that $\mu^{(r)}$ must coincide with $D^{r} \mu$ in the sense of distributions. In particular, therefore, $D^{m} \mu \in L^{p}$. Finally, the relation $\mu_{i} \rightarrow \mu$ weakly in $L^{p}$ combines with equation (4.2) to yield (4.1).

The excluded cases. (a) The case $m=\infty$. This is covered by trifling modifications in the above proof. The condition that $D^{r} \mu \in L^{p}$ for every (finite) $r$ implies that $\mu \in C^{(\infty)}$ (cf. [3, Tome I, p. 55]), but appeal to this theorem could be avoided because now (4.2) shows that the functions $\mu_{i}$ fall into a bounded subset of $C^{(\infty)}$ and, by Ascoli's theorem, these bounded subsets are all relatively compact in $C^{(\infty)}$. On the other hand, this type of proof could be entirely 
replaced by more direct arguments appropriate to this special case. To begin with, a very simple argument shows that, if $G$ is compact and $E$ contains all characters of $G$, then any $u \in L_{t}(E, F)$ corresponds to a factor function of class $(E, F)$ which is itself uniquely determined by the equations

$$
u(\hat{x})=\phi(\hat{x}) \cdot x
$$

for all $\hat{x} \in \hat{G}$. In the second place, if $G$ is the circle group and $F$ is $C^{(\infty)}$, it is equally simple to show that $\phi$ is a factor function if and only if it is "rapidly decreasing" in the sense that

$$
\lim _{\hat{x} \rightarrow \infty}|\hat{x}|^{k}|\phi(\hat{x})|=0
$$

for each $k=0,1,2, \ldots$, and that this in turn is equivalent to the condition that $\phi=\hat{\mu}$ for some $\mu \in C^{(\infty)}$.

(b) The case $p=\infty$. In view of remarks (a), one may assume that $m$ is finite. A preliminary reduction allows one to assume that $m=0$. So we have the representation problem for mappings $u \in L_{t}\left(L^{\infty}, C\right)$. The arguments of Theorem 4 break down for the familiar reason that $L^{1}$ is not reflexive. However, it is obvious that the condition $\mu \in L^{1}$ is sufficient in order that (4.1) shall define $u$ as a member of $L_{t}\left(L^{\infty}, C\right)$. The difficulty in proving the converse lies solely in showing that

$$
u(k * f)=u(k) * f
$$

is valid for any two functions $k, f \in L^{\infty}$. This is obviously true whenever $u$ is derived from a factor function of class $\left(L^{\infty}, C\right)$; it is also true more generally whenever $u$ commutes with translations and is continuous for the weak topologies $\sigma\left(L^{\infty}, L^{1}\right)$ and $\sigma\left(C, M^{1}\right)$.

Assuming that $(4.3)$ is true, we can prove the representation

$$
u(f)=\mu * f
$$

for some $\mu \in L^{1}$ depending solely on $u$, provided the compact $G$ satisfies the first axiom of countability. In fact, let $\left(k_{i}\right)$ be an "approximate identity" formed of a denumerable sequence of functions in $L^{\infty}$. Then $k_{i} * f \rightarrow f$ weakly in $L^{\infty}$, and hence,

$$
u\left(k_{i} * f\right) \rightarrow u(f)
$$


for $\sigma\left(C, M^{1}\right)$. On the other hand

$$
u\left(k_{i} * f\right)=\mu_{i} * f
$$

where $\mu_{i}=u\left(k_{i}\right) \in C$. It follows that the sequence $\left(\mu_{i}\right)$ is a weak Cauchy sequence in $L^{1}$, hence has a weak limit $\mu \in L^{1}$. But then

$$
u(f)=\lim _{i} u\left(k_{i} * f\right)=\lim _{i}\left(\mu_{i} * f\right)=\mu * f,
$$

identification being made in the pointwise sense. This completes the proof and incidentally solves the factor problem for $\left(L^{\infty}, C\right)$.

\section{REFERENCES}

1. A. Grothendieck, Sur la complétion du dual d'un espace localement convexe, C. R. (Paris), 230, (1950), 605-606.

2. E. Hille, Functional analysis and semigroups, Amer. Math. Soc. Coll. Publications XXXI (1948).

3. S. Kaczmarz and H. Steinhaus, Orthogonalreihen, Warsaw (1935).

4. L. Schwartz, Théorie des distributions, Tomes I, II, Paris (1950, 1951).

5. A. Zygmund, Trigonometrical series, Warsaw (1935).

\section{BIR KBECK COLLEGE}

UNIVERSITY OF LONDON 


\section{PACIFIC JOURNAL OF MATHEMATICS}

\section{EDITORS}

\author{
H.L. ROYDEN \\ Stanford University \\ Stanford, California \\ E. HEWITT \\ University of Washington \\ Seattle 5, Washington
}

\author{
R. P. DILWORTH \\ California Institute of Technology \\ Pasadena 4, California \\ * Alfred Horn \\ University of California \\ Los Angeles 24, California
}

\section{ASSOCIATE EDITORS}

\section{H, BUSEMANN \\ HERBERT FEDERER}

MARSHALL HALL

\section{P.R. HALMOS \\ HEINZ HOPF}

ALFRED HORN

\author{
R.D. JAMES \\ BØRGE JESSEN \\ PAUL LÉVY
}

GEORGE PÓLYA

J.J. STOKER

KOSAKU YOSIDA

\section{SPONSORS}

UNIVERSITY OF BRITISH COLUMBIA

UNIVERSITY OF SOUTHERN CALIFORNIA

CALIFORNIA INSTITUTE OF TECHNOLOGY

UNIVERSITY OF CALIFORNIA, BER KELEY

STANFORD RESEARCH INSTITUTE

STANFORD UNIVERSITY

UNIVERSITY OF CALIFORNIA, DAVIS

UNIVERSITY OF. UTAH

UNIVERSITY OF CALIFORNIA, LOS ANGELES

WASHINGTON STATE COLLEGE

UNIVERSITY OF CALIFORNIA, SANTA BARBARA

UNIVERSITY OF WASHINGTON

MONTANA STATE UNIVERSITY

UNIVERSITY OF NEVADA

OREGON STATE COLLEGE

AMERICAN MATHEMATICAL SOCIETY

HUGHES AIRCRAFT COMPANY

UNIVERSITY OF OREGON

SHELL DEVELOPMENT COMPANY

\section{UNIVERSTTY OF SOUTHERN CALIFORNIA}

Mathematical papers intended for publication in the Pacific Journal of Mathematics should be typewritten (double spaced), and the author should keep a complete copy. Manuscripts may be sent to any of the editors. Manuscripts intended for the outgoing editors should be sent to their successors. All other communications to the editors should be addressed to the managing editor, Alfred Horn, at the University of California Los Angeles 24, California.

50 reprints of each article are furnished free of charge; additional copies may be obtained at cost in multiples of 50 .

The Pacific Journal of Mathematics is published quarterly, in March, June, September, and December. The price per volume (4 numbers) is $\$ 12.00$; single issues, $\$ 3.50$; back numbers (Volumes $1,2,3$ ) are available at $\$ 2.50$ per copy. Special price to individual faculty members of supporting institutions and to individual members of the American Mathematical Society: $\$ 4.00$ per volume; single issues, $\$ 1.25$.

Subscriptions, orders for back numbers, and changes of address should be sent to the publishers, University of California Press, Berkeley 4, California.

Printed at Ann Arbor, Michigan. Entered as second class matter at the Post Office, Berkeley, California.

* During the absence of E.G. Straus.

UNIVERSITY OF CALIFORNIA PRESS - BERKELEY AND LOS ANGELES 


\section{Pacific Journal of Mathematics}

\section{Vol. 5, No. $3 \quad$ November, 1955}

Nesmith Cornett Ankeny and S. Chowla, On the divisibility of the class number of quadratic fields ............................. 321

Cecil Edmund Burgess, Collections and sequences of continua in the plane ........................................ 325

Jane Smiley Cronin Scanlon, The Dirichlet problem for nonlinear elliptic equations....................................... 335

Arieh Dvoretzky, A converse of Helly's theorem on convex sets ......... 345

Branko Grünbaum, On a theorem of L. A. Santaló................ 351

Moshe Shimrat, Simple proof of a theorem of P. Kirchberger .......... 361

Michael Oser Rabin, A note on Helly's theorem . ................... 363

Robert E. Edwards, On factor functions . . ................... 367

Robert E. Edwards, On certain algebras of measures ............... 379

Harley M. Flanders, Methods in affine connection theory.............. 391

Alfred Huber, The reflection principle for polyharmonic functions ........ 433

Geoffrey Stuart Stephen Ludford, Generalised Riemann invariants ....... 441

Ralph Gordon Selfridge, Generalized Walsh transforms............. 451 